\title{
Les jeunes de 15 ans sont-ils devenus plus « verts »?
}

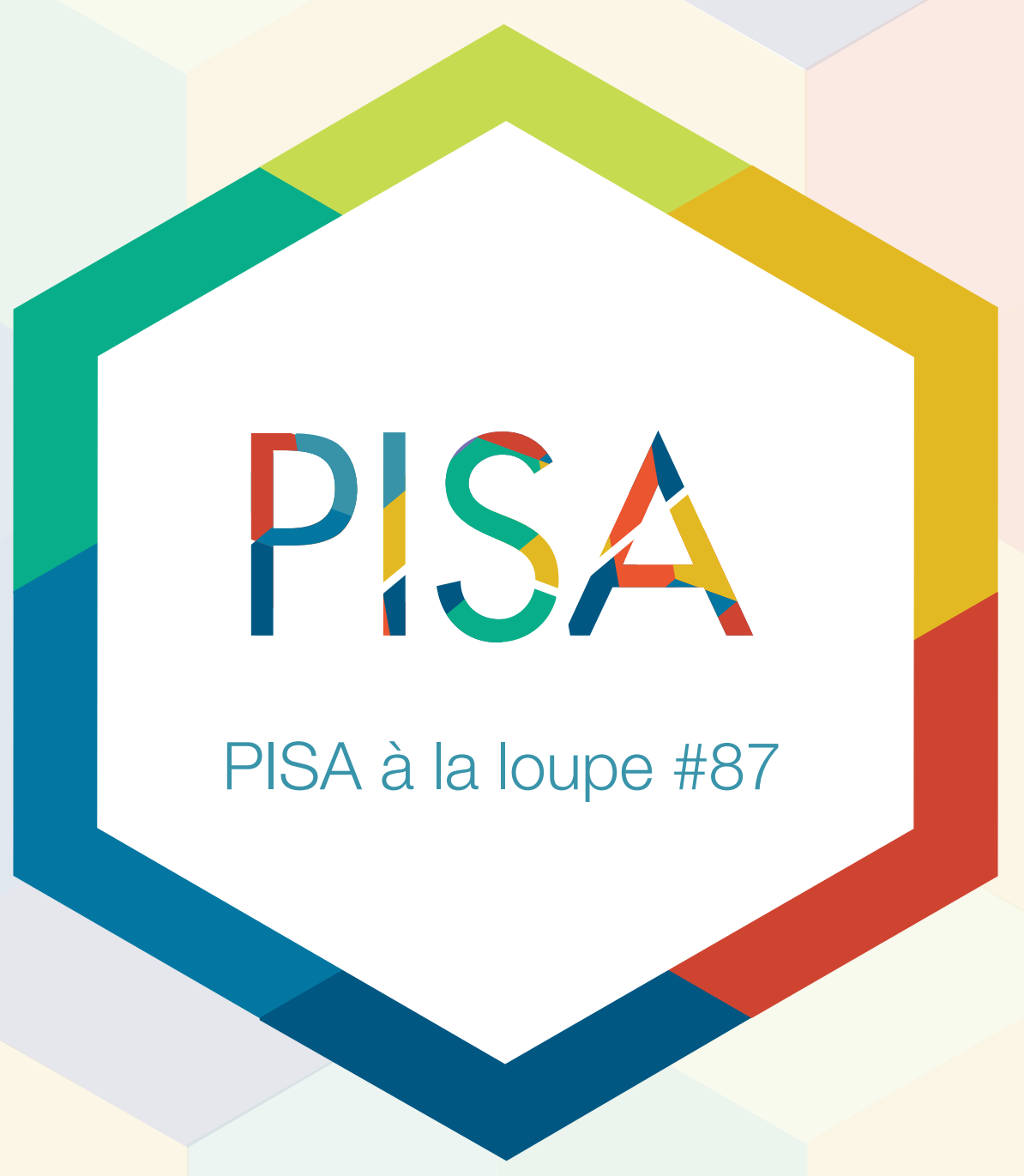




\section{Les jeunes de 15 ans sont-ils devenus plus « verts »?}

- Durant les neuf années écoulées entre 2006 de 2015, le pourcentage d'élèves se disant informés ou bien informés au sujet des problèmes environnementaux a connu une hausse modérée, en moyenne, dans les pays de l'OCDE. Parallèlement, les élèves ne sont pas devenus plus optimistes quant à la possibilité de résoudre ces problèmes.

- Les élèves sensibilisés aux problèmes environnementaux sont plus pessimistes à l'égard de l'avenir de la planète. Les élèves de 15 ans se disant informés de l'augmentation des gaz à effet de serre sont ainsi 43 \% plus susceptibles d'estimer que ce problème s'aggravera au cours des 20 prochaines années.

- Rares sont les caractéristiques des élèves et des établissements qui sont liées positivement à la fois à la sensibilisation aux problèmes environnementaux et à l'optimisme à l'égard de ces derniers, mais les plus importantes sont le nombre d'activités scientifiques auxquelles participent les élèves et leur exposition à un enseignement fondé sur une démarche d'investigation.

Notre planète a connu bien des transformations (négatives) depuis plus de 10 ans, lorsque l'enquête PISA interrogeait les élèves sur l'environnement. Augmentation de la température mondiale, fonte des glaciers, récifs coralliens toujours plus menacés, hausse d'environ 3 centimètres du niveau de la mer, accumulation des déchets dans les océans, sans compter les catastrophes provoquées par l'homme, comme la marée noire suite à l'explosion de la plateforme Deepwater Horizon ou encore la catastrophe nucléaire de Fukushima : autant de périls qui mettent encore plus en danger la santé déjà fragile de notre planète.

À travers des initiatives nationales et internationales, telles que la Conférence et l'Accord de Paris sur le climat - aussi connus sous le nom de COP21 -, les gouvernements s'efforcent de coordonner leurs efforts pour protéger l'environnement ; toutefois, tant que la société n'aura pas pleinement pris conscience des conséquences de l'inaction, le coût de l'action pourra encore sembler trop élevé. Les élèves sont-ils mieux informés au sujet des problèmes environnementaux? Les jeunes de 15 ans sont-ils aujourd'hui plus optimistes quant à l'avenir de la planète? Qui sont les élèves les plus sensibilisés aux problématiques environnementales ?

\section{Sensibilisation aux problèmes environnementaux et optimisme}

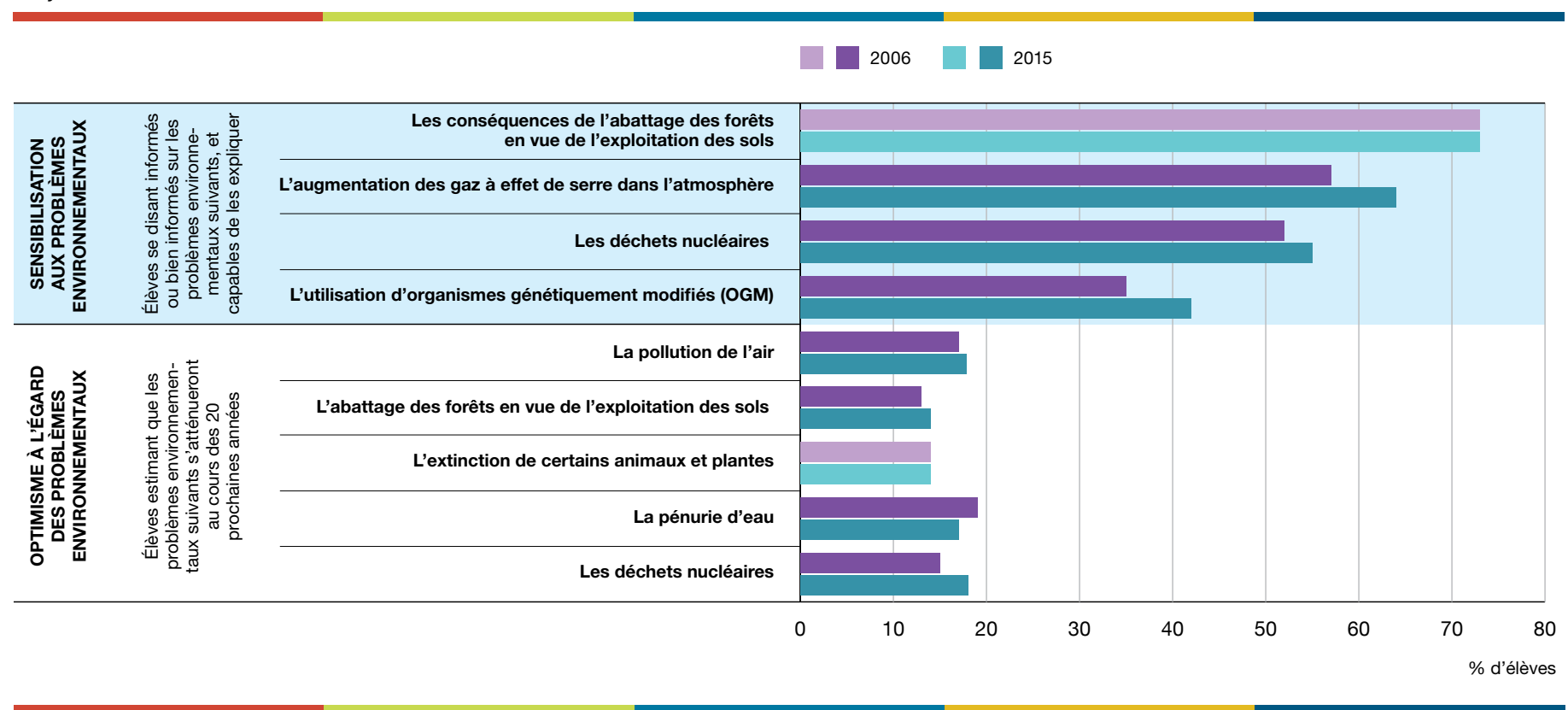

Remarques : La Lituanie est devenue membre de l'OCDE le 5 juillet 2018 et n'est pas incluse dans la moyenne OCDE. Les différences statistiquement significatives entre 2006 et 2015 sont indiquées dans une couleur plus foncée.

Source : OCDE, Base de données PISA. 
Évolution entre 2006 et 2015 de la sensibilisation aux problèmes environnementaux et de l'optimisme à leur égard

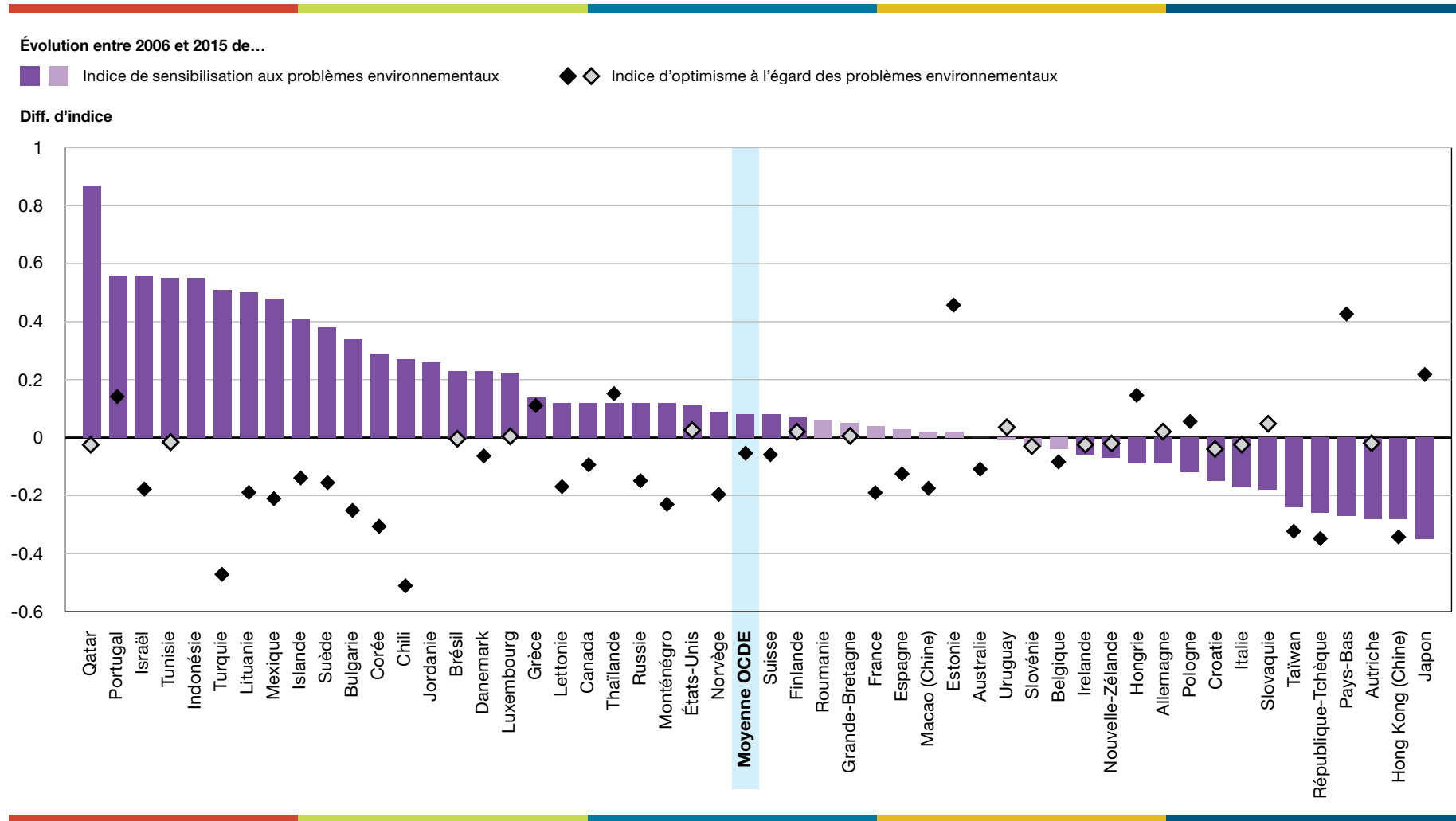

Remarques : Les différences statistiquement significatives entre 2006 et 2015 sont indiquées dans une couleur plus foncée.

La Lituanie est devenue membre de l'OCDE le 5 juillet 2018 et n'est pas incluse dans la moyenne OCDE.

Les indices de sensibilisation aux problèmes environnementaux et d'optimisme à leur égard mesurent respectivement le degré de sensibilisation et d'optimisme des élèves à l'égard des problèmes environnementaux. Ils sont mis à l'échelle à l'aide de la théorie de la réponse aux items et ont une moyenne de 0 et un écart-type de 1 dans les pays de l'OCDE.

Source : OCDE, Base de données PISA.

\section{Les jeunes de 15 ans sont de plus en plus sensibilisés aux problèmes environnementaux.}

L'enquête PISA a interrogé les élèves de 15 ans sur leur degré d'information concernant un certain nombre de problèmes environnementaux, comme la pollution de l'air, la pénurie d'eau ou les déchets nucléaires. Durant les neuf années écoulées entre 2006 et 2015, et pour la plupart des problèmes environnementaux cités dans ces deux éditions de l'enquête PISA, le pourcentage d'élèves se disant informés (" Je vois de quoi il s'agit et je pourrais expliquer cela dans les grandes lignes") ou bien informés (" Je connais ce sujet et je pourrais clairement expliquer de quoi il s'agit ") a connu une hausse modérée, en moyenne, dans les pays de l'OCDE. Le pourcentage d'élèves se disant informés de l'augmentation des gaz à effet de serre dans l'atmosphère a ainsi augmenté, passant de 57 \% en 2006 à 64 \% en 2015 ; une hausse similaire s'observe au sujet de l'utilisation d'organismes génétiquement modifiés (OGM).

Cette amélioration globale de la sensibilisation des élèves aux problèmes environnementaux résulte en grande partie de la hausse, en Israël, au Mexique, au Portugal et en Turquie, du pourcentage d'élèves se disant informés à ce sujet. Dans les pays partenaires de I'OCDE, des augmentations similaires s'observent en Indonésie, au Qatar et en Tunisie. D'un autre côté, c'est en Autriche, à HongKong (Chine), en Italie, au Japon, aux Pays-Bas et en République tchèque que la sensibilisation des élèves aux problèmes environnementaux a connu le recul le plus marqué, même s'il reste modéré.

\section{Cependant, les élèves ne sont pas devenus plus optimistes à l'égard des problèmes environnementaux.}

En moyenne, dans les pays de l'OCDE, le pourcentage d'élèves se disant optimistes quant à l'avenir de la planète - ceux estimant que les problèmes environnementaux s'atténueront au cours des 20 prochaines années - est toutefois resté relativement stable durant cette même période. En 2015, les jeunes de 15 ans étaient légèrement plus optimistes que leurs homologues en 2006 à l'égard des 
Les élèves sensibilisés aux problèmes environnementaux sont-ils plus pessimistes concernant l'avenir de la planète?

Moyenne OCDE

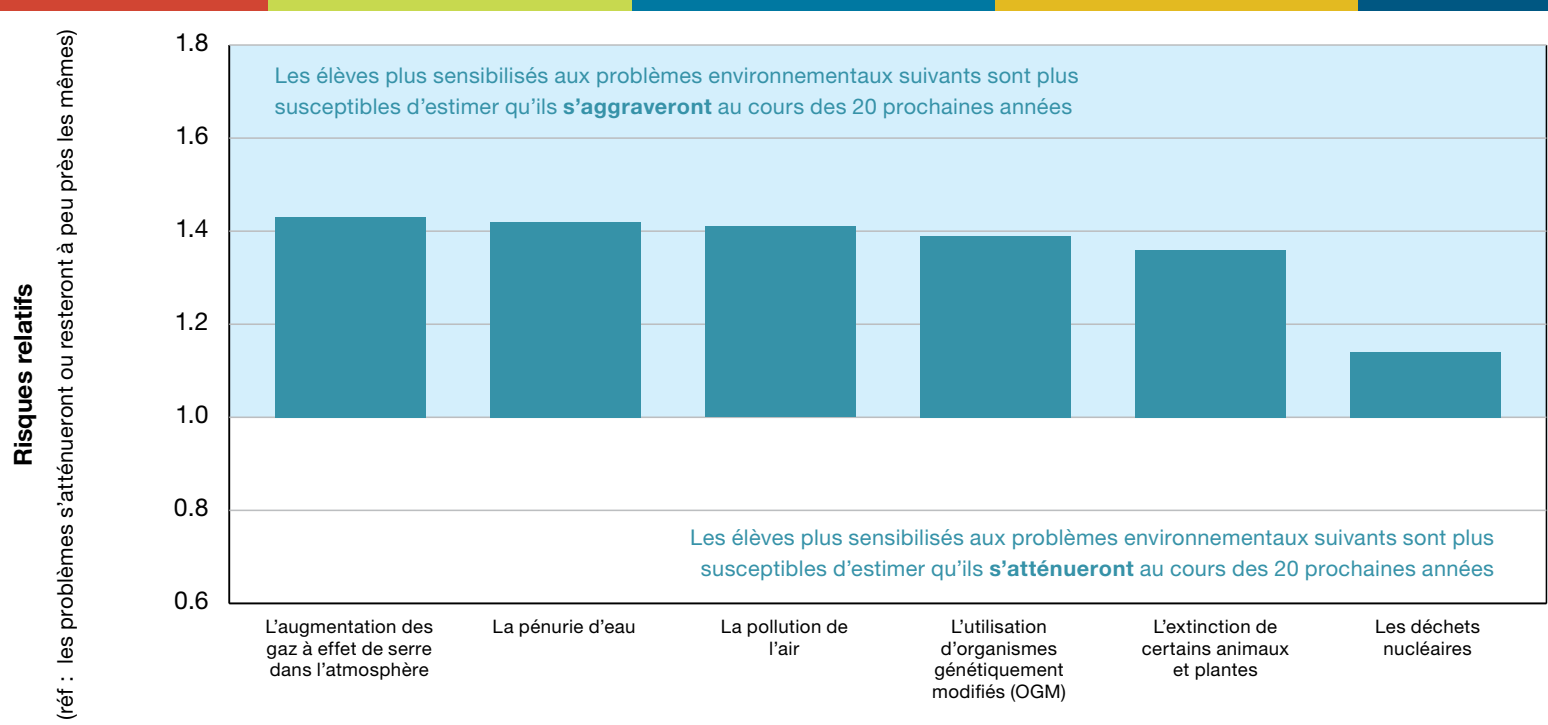

Les élèves se disent informés des problèmes environnementaux suivants et capables de les expliquer ${ }^{1}$

1. Inclut les élèves ayant choisi les réponses suivantes : « Je vois de quoi il s'agit et je pourrais expliquer cela dans les grandes lignes » ou « Je connais ce sujet et je pourrais clairement expliquer de quoi il s'agit ".

La catégorie de référence inclut les élèves ayant choisi les réponses suivantes : « Je n'en ai jamais entendu parler » ou " J'en ai entendu parler mais je serais incapable d'expliquer ce que c'est exactement ".

Remarques : La Lituanie est devenue membre de l'OCDE le 5 juillet 2018 et n'est pas incluse dans la moyenne OCDE.

Toutes les valeurs sont statistiquement significatives.

Source : OCDE, Base de données PISA.

problèmes liés à l'abattage des forêts, aux déchets nucléaires et à la pollution de l'air, mais plus pessimistes concernant l'accès à l'eau dans le futur. La plus forte avancée de l'optimisme à l'égard des problèmes environnementaux s'observe en Estonie, au Japon et aux Pays-Bas, et celle du pessimisme, en Bulgarie, au Chili, en Corée, à Hong-Kong (Chine), en République tchèque, au Taipei chinois et en Turquie. II est intéressant de noter que dans la majorité des systèmes d'éducation où la sensibilisation des élèves aux problèmes environnementaux s'est améliorée entre 2006 et 2015, comme en Bulgarie, au Chili, en Corée, en Islande, en Israël, en Lituanie, au Mexique, en Suède et en Turquie, leur optimisme concernant l'avenir de la planète a quant à lui diminué.

\section{Les élèves sensibilisés aux problèmes environnementaux sont plus pessimistes quant à l'avenir de la planète.}

Rien de très surprenant à ce qu'une plus grande sensibilisation aux problèmes environnementaux ne se traduise pas par un plus grand optimisme, étant donné que les élèves se disant informés de ces problèmes sont considérablement plus susceptibles d'estimer que ceux-ci s'aggraveront dans le futur. Ainsi, les élèves se disant informés de l'augmentation des gaz à effet de serre, de la pénurie d'eau et de la pollution de l'air sont environ 40 \% plus susceptibles d'estimer que ces problèmes s'aggraveront au cours des 20 prochaines années.

\section{Nombre de caractéristiques des élèves et des établissements sont positivement} liées à la sensibilisation aux problèmes environnementaux, mais seules quelquesunes le sont aussi à l'optimisme à l'égard de ces problèmes.

En moyenne, dans les pays de l'OCDE, les élèves se disant plus sensibilisés aux problèmes environnementaux présentent de nombreuses caractéristiques communes. Les élèves issus d'un milieu socio-économique favorisé sont par exemple considérablement plus susceptibles que leurs pairs défavorisés de se dire informés au sujet des problèmes environnementaux. Les élèves ayant le goût 
Optimisme à l'égard des problèmes environnementaux Sensibilisation aux problèmes environnementaux

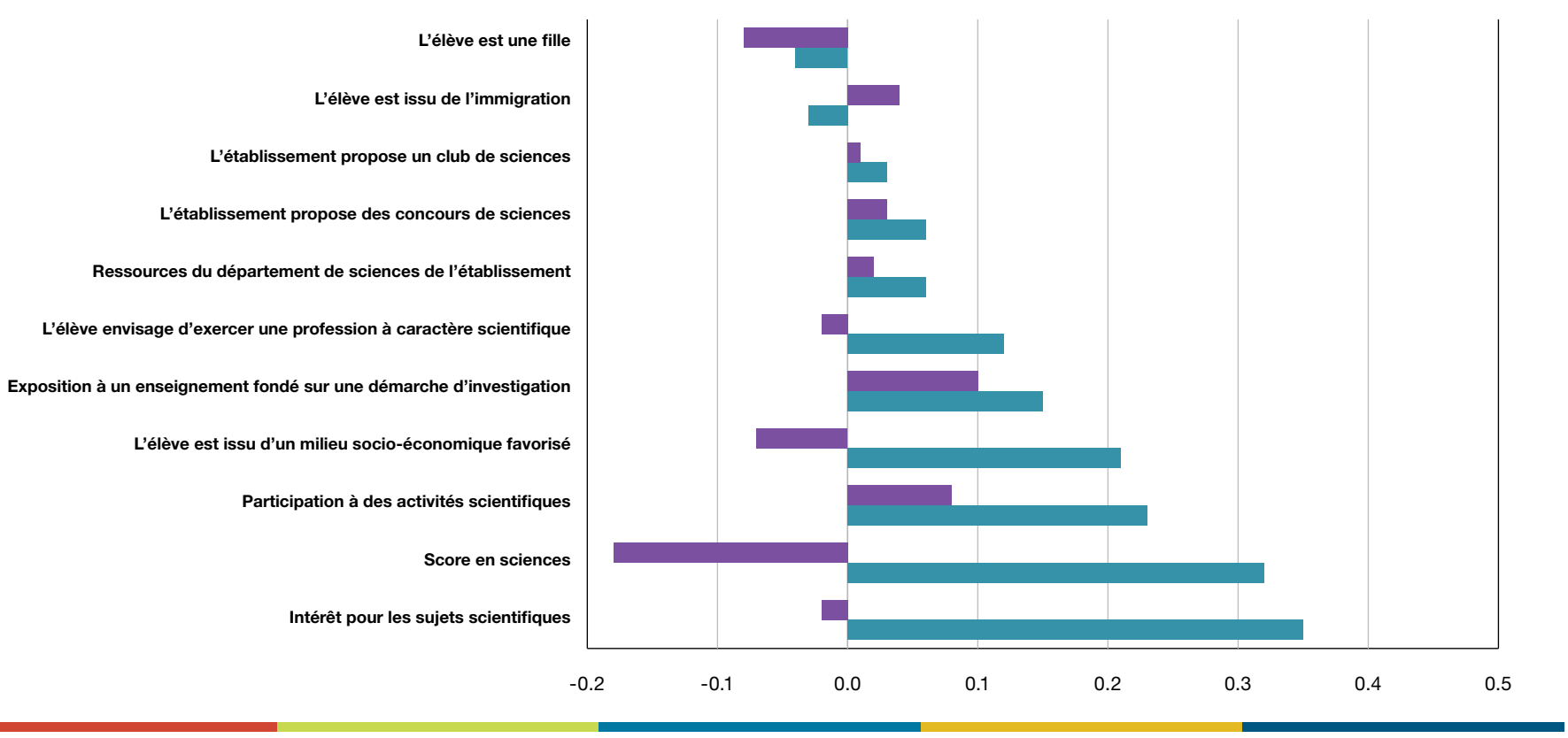

Remarques : La Lituanie est devenue membre de l'OCDE le 5 juillet 2018 et n'est pas incluse dans la moyenne OCDE. Tous les coefficients de corrélation sont statistiquement significatifs. Source : OCDE, Base de données PISA.

des sciences - soit les élèves très performants qui participent à des activités scientifiques, envisagent d'exercer une profession à caractère scientifique et s'intéressent aux sujets scientifiques - font part d'une plus grande sensibilisation aux problèmes environnementaux. Les élèves tendent aussi à être sensibilisés à ces problèmes lorsqu'ils font part d'une plus forte exposition à un enseignement fondé sur une démarche d'investigation et, dans une moindre mesure, lorsque le département scientifique de leur établissement est doté des ressources adéquates et propose des activités scientifiques, notamment des concours et un club de sciences.

Des recherches antérieures ont tenté de déterminer les facteurs incitant les citoyens à adopter un comportement respectueux de l'environnement - c'est-à-dire des actions visant à minimiser l'impact négatif des hommes sur l'environnement. Parmi les conclusions ainsi mises au jour, il ressort que la sensibilisation aux problèmes environnementaux est certes essentielle, mais ne suffit pas à elle seule à encourager l'action (voir, par exemple, les travaux souvent cités d'Anja Kollmuss et de Julian Agyeman). Les personnes estimant que ces problèmes s'aggraveront peuvent ainsi considérer que tout ce qu'ils pourront entreprendre sera insuffisant ou inutile, bien qu'un optimisme aveugle puisse aussi être source d'inaction. La question est donc la suivante : comment amener les élèves sur la voie d'un optimisme éclairé ? Si l'enquête PISA ne peut démontrer les liens de causalité, seuls deux facteurs présentent une forte association à la fois avec la sensibilisation aux problèmes environnementaux et la conviction qu'ils pourront s'atténuer au cours des 20 prochaines années : le nombre d'activités scientifiques auxquelles participent les élèves et leur exposition à un enseignement fondé sur une démarche d'investigation.

\section{Pour conclure}

La plupart des gens s'accordent à reconnaître la dégradation de l'environnement ces dernières décennies, même si le débat demeure sur l'ampleur et les conséquences de ces changements. II existe heureusement quantité d'initiatives publiques et privées pour protéger l'environnement, et les élèves du monde entier sont de plus en plus sensibilisés aux problèmes environnementaux les plus importants qui touchent aujourd'hui notre planète. Toutefois, les élèves ne se montrent pas plus optimistes quant à l'avenir. Si nous souhaitons préserver l'environnement pour les générations à venir, il est essentiel de sensibiliser davantage les élèves aux problèmes environnementaux afin qu'ils mettent ces connaissances au service de modes de vie durables, de la réduction des coûts de l'action et de la recherche de solutions novatrices. 


\section{Pour tout complément d'information}

Contact : Alfonso Echazarra (alfonso.echazarra@oecd.org)

Le mois prochain : Performance et climat des établissements : quel lien avec l'expérience des enseignants ?

Cet ouvrage est publié sous la responsabilité du Secrétaire général de l'OCDE. Les opinions et les interprétations exprimées ne reflètent pas nécessairement les avis des pays membres de l'OCDE.

Ce document, ainsi que les données et cartes qu'il peut comprendre, sont sans préjudice du statut de tout territoire, de la souveraineté s'exerçant sur ce dernier, du tracé des frontières et limites internationales, et du nom de tout territoire, ville ou région.

Les données statistiques concernant Israël sont fournies par et sous la responsabilité des autorités israéliennes compétentes. L'utilisation de ces données par l'OCDE est sans préjudice du statut des hauteurs du Golan, de Jérusalem-Est et des colonies de peuplement israéliennes en Cisjordanie aux termes du droit international.

Ce texte est disponible sous licence Attribution - Pas d'Utilisation Commerciale - Partage dans les Mêmes Conditions 3.0 Organisations Internationales (CC BY-NC-SA 3.0 IGO). Pour toute information spécifique quant à l'étendue et aux termes de la licence ainsi que d'une possible utilisation commercial de ce texte et pour toute usage de données PISA, prière de consulter les Conditions d'utilisation à http://www.oecd.org/fr/conditionsdutilisation. 